\title{
Unexpected Etiology of Pure Macroscopic Hematuria: Emphysematous Cystitis
}

\author{
Mithat Ekşi, İsmail Evren, Feyzi Arda Atar, Volkan Tuğcu \\ Department of Urology, Bakırköy Dr. Sadi KonukTraining and Research Hospital, İstanbul, Turkey
}

\begin{abstract}
Introduction: Emphysematous cystitis (EC) is a rare type of urinary tract infection characterized by the presence of gas in the bladder lumen. It is usually encountered in older women with uncontrolled diabetes mellitus (DM). Patients usually present with dysuria, hematuria, suprapubic pain, and less frequently, with pneumaturia. For definitive diagnosis, pathological examination is required but in most cases, computed tomography $(\mathrm{CT})$ is adequate. With appropriate antibiotherapy and drainage; EC can be managed.
\end{abstract}

Case Report: A 78-year-old woman who presented presented with hematuria was diagnosed with EC via CT scan. The patient was treated with antibiotherapy and placement of urethral Foley catheter.

Conclusion: In the diagnosis and treatment of urinary tract infection in elderly women with uncontrolled DM, EC should be kept in mind because of its complications, and for suspicious cases, CT should be performed.

Keywords: Cystitis, diabetes, emphysematous, hematuria

Received: 21.04.2016 Accepted: 26.05.2016 Available Online Date: 24.10.2016

\section{Introduction}

Emphysematous cystitis (EC) is a rare type of urinary tract infection and is characterized by the presence of gas in the bladder lumen. It is usually encountered in older women with uncontrolled diabetes mellitus (DM) or immune deficiency. The process has a broad spectrum, and clinic presentation can be variable: EC can be asymptomatic or even mortal. Patients usually present with dysuria, hematuria, suprapubic pain, and less frequently with pneumaturia. Early diagnosis and treatment prevents more complicated conditions like bladder necrosis, emphysematous pyelonephritis, and/or urosepsis. For definitive diagnosis, pathological examination is required, but in most cases, computed tomography (CT) is adequate. With appropriate antibiotherapy and drainage, EC can be managed (1, 2).

Here we present the case of an elderly patient with uncontrolled DM who has EC, with hematuria being the only symptom.

\section{Case Report}

A 78-year-old female patient was taken to the emergency room with pure macroscopic hematuria. The patient had Alzheimer's disease, type 2 DM, and hypertension. Patient history revealed no alcohol consumption or smoking. Patient had been taking a daily dose of $100 \mathrm{mg}$ acetylsalicylic acid.

Patient's vital signs were stable and no pathological findings were observed on physical examination, and the patient had a body mass index of 27.5. White blood cell count was $6700 / \mathrm{mm}^{3}$ and creatinine level was $0.88 \mathrm{mg} / \mathrm{dL}$. C-reactive protein level was $3 \mathrm{mg} /$ $\mathrm{dL}(\mathrm{n}<0.8)$. Pre-prandial blood glucose was $138 \mathrm{mg} / \mathrm{dL}$ and the value of HbA1c was 7.5\%. No other altered biochemical parameter was observed. Urinalysis showed >100 red blood cell/high power field, 100 white blood cell/ high power field and some bacteria.

Both kidneys were observed to be normal on ultrasonography (USG). Due to the suspicious ultrasonographic findings such as echogenicity at the bladder wall, an abdominal CT was performed. Intraluminal gas was observed on CT (Figure 1). A Foley catheter was then placed for bladder drainage. Intravenous cefuroxime treatment was administered and intravenous insulin infusion was started for hyperglycemia. 

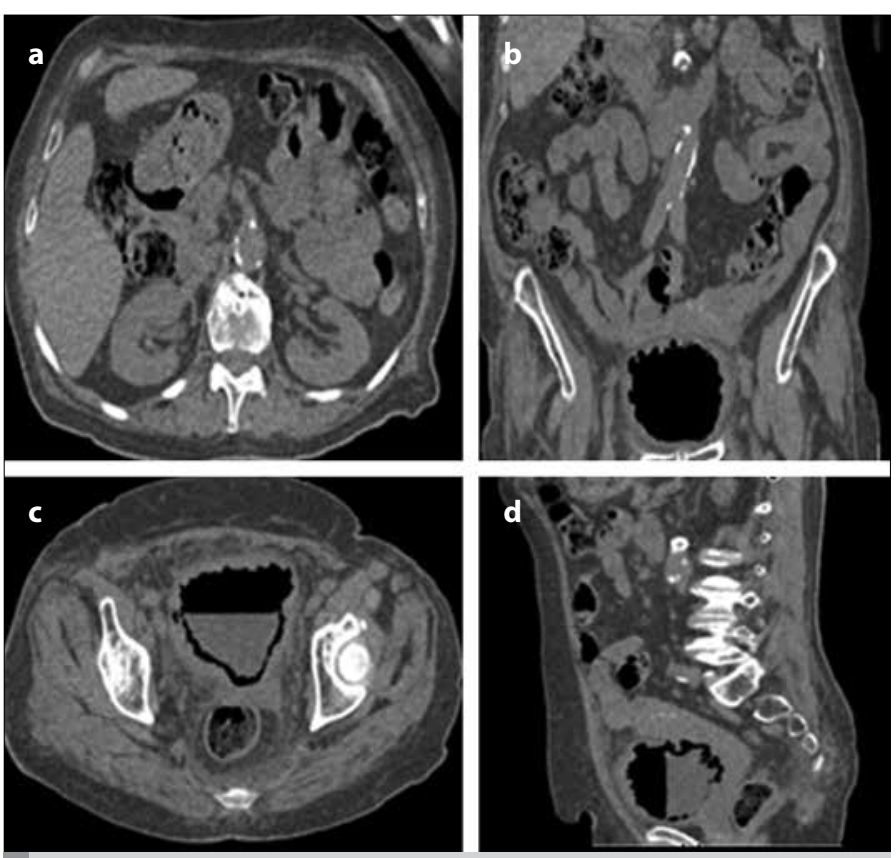

Figure 1. a-d. (a) Normal kidneys. Gas in the bladder lumen. (b) coronal plane (c) axial plane (d) sagittal plane
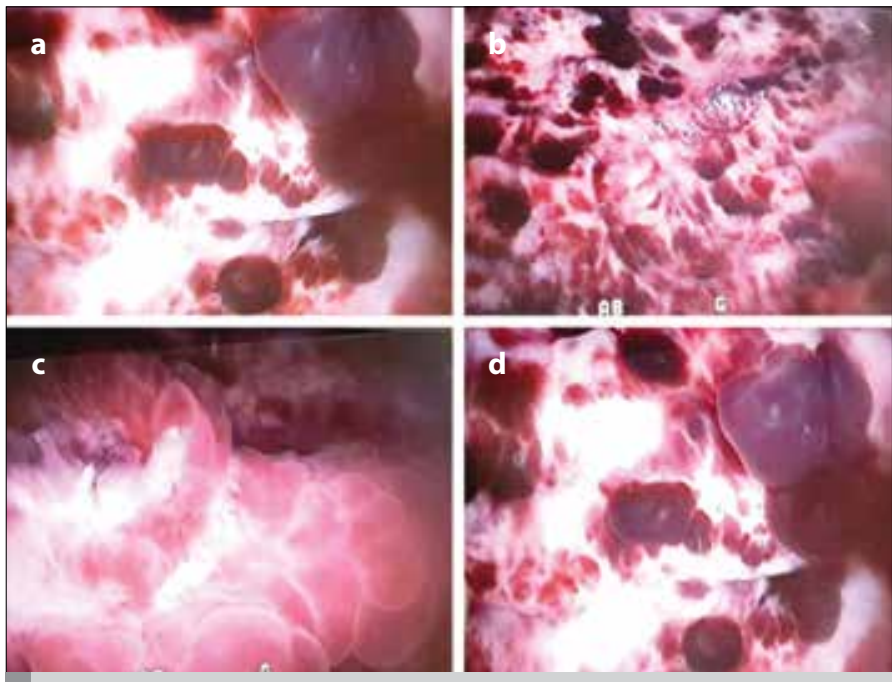

Figure 2. a-d. Cystoscopic images. (a) Cystic lesions. (b) Hemorrhagia from the mucosa. (c) Papillary-like lesions. (d) Abnormal mucosa and hemorrhagic cystic lesions

Hematuria regressed with antibiotic treatment and drainage. The urine culture showed Escherichia coli infection sensitive to cefuroxime. The patient was discharged with oral cefuroxime treatment on day 3 and catheter was removed on day 5. Neither fistula nor diverticulum was observed during cystoscopy performed on day 8. Multiple hemorrhagic and bullous papillary lesions were observed (Figure 2).

Intraluminal gas collection regression was revealed at the Control CT which was performed 2 weeks after admission. The patient was asymptomatic and the urine culture was sterile on follow-up.

Written informed consent was received from patient.

\section{Discussion}

Gas-forming infections should be considered risky due to high possibility of complications. EC is observed more frequently in patients with DM because of the effects of DM on the urinary system, bladder dysfunction caused by neuropathy, and suppressed immune response caused by uncontrolled blood glucose levels $(1,3)$.

The mean age for patients with EC was reported 66 years in the literature (1). The male/female ratio is $1 / 2$ (4). Seven percent of patients are reported to be asymptomatic (1). Non-specific symptoms such as abdominal pain, nausea, vomiting, and fever were reported (2). Abdominal pain was the most common symptom (4). The typical symptoms of urinary tract infection were encountered in 53\% percent of patients (2). USG and magnetic resonance imaging are usually inadequate. Cystoscopy alone is usually not adequate but reveals submucosal emphysema and bladder outlet obstruction (1). CT has the best specificity and sensitivity $(1,2,4)$. In our case, the only pathologic finding was hematuria. The patient was incidentally diagnosed via CT, which was performed due to findings on USG.

E. coli is the most frequently isolated bacteria on urine culture (58\% of patients). Furthermore, uropathogens including Klebsiella pneumoniae (21\%), Enterobacter spp. (7\%), Clostridium perfringens (6\%), Proteus spp. (3\%), Staphylococcus spp., Streptococcus spp., and Candida albicans (4\%) have also been reported to be isolated from urine culture (1). In our case, E. coli was isolated from urine culture, which was sensitive for cefuroxime treatment. The patient was treated for the first 3 days with intravenous cefuroxime and for the next 7 days with oral cefuroxime. Grupper et al. recommended 10 days of antibiotics and at least a 7-day hospital stay (2). In our case, due to the regression of the infection and hematuria, the patient was discharged on day 3 with a urinary catheter (2).

$\mathrm{CT}$ is not a routine examination at the emergency clinic for urinary tract infections, but in cases of suspicious findings on USG such as echogenicity on bladder wall, acoustic imaging, especially for patients who have immune deficiency or uncontrolled DM, ES should be kept in mind and CT should be performed if necessary. CT has a high sensitivity and specificity for the elimination of vesicocolic fistula, intraabdominal abscess, or neoplasm and observing of intramural or paravesical gas (4).

Treatment suggestions include antibiotics, urinary drainage, and hyperglycemia control. In the literature, there are some cases that were treated with hyperbaric oxygen and showed rapid response (5). When medical treatments fail or necrotizing tissue is detected, surgical procedures such as cystectomy or debridement may be considered (6). Mortality rate with ES is 7\%, but this number can be more if the upper urinary system is affected (1).

\section{Conclusion}

When elderly patients with urinary tract infections who have uncontrolled DM are presented with hematuria, in case of suspicious ultrasonographic findings, EC should be kept in mind and a CT should be performed. EC is a deadly disease, but has good prognosis on early diagnosis, drainage, and appropriate treatment. 
Informed Consent: Written informed consent was obtained from patient who participated in this case.

Peer-review: Externally peer-reviewed.

Author contributions: Concept - M.E., I.E., F.A.A.; Design - F.A.A., I.E., M.E.; Supervision - F.A.A., I.E., V.T.; Resource - M.E., I.E.; Materials - M.E., F.A.A.; Data Collection and/or Processing - M.E., V.T.; Analysis and/or Interpretation - F.A.A., I.E., V.T.; Literature Search - M.E.; Writing - M.E., V.T.; Critical Reviews - I.E., F.A.A., i.E.

Conflict of Interest: No conflict of interest was declared by the authors.

Financial Disclosure: The authors declared that this study has received no financial support

\section{References}

1. Thomas AA, Lane BR, Thomas AZ, Remer EM, Campbell SC, Shoskes DA. Emphysematous cystitis: a review of 135 cases. BJU Int 2007; 100: 17-20. [CrossRef]

2. Grupper M, Kravtsov A, Potasman I. Emphysematous cystitis: illustrative case report and review of the literature. Medicine (Baltimore) 2007; 86: 47-53.

3. Akan O. Rare infections in diabetes. Turkiye Klinikleri J Endocrin-Special Topics 2008; 1: 64-70.

4. Tagowski M, Kamusella $P$, Andresen $R$. A rare cause of abdominal pain: emphysematous cystitis. Rofo 2013; 184: 998-9. [CrossRef]

5. McCabe JB, Mc-Ginn Merritt W, Olsson D, Wright V, Camporesi EM. Emphysematous cystitis: rapid resolution of symptoms with hyperbaric treatment: a case report. Undersea Hyperb Med 2004; 31: 281-4.

6. Perlmutter AE, Mastromichaelis M, Zaslau S. Emphysematous cystitis: a case report and literature review. WV Med J 2004; 100: 232-3. 